\title{
Percepciones de docentes y directores sobre los factores que promueven u obstaculizan la educación ambiental en escuelas del Área Metropolitana de Buenos Aires
}

\author{
Perceptions of teachers and directors about the factors \\ that promote or constrain environmental education \\ in schools of Metropolitan Area of Buenos Aires, Argentina
}

\author{
Mariana Saidón $^{1}$. Julieta Andrea Claverie ${ }^{2}$
}

\begin{abstract}
Resumen: El trabajo aborda las percepciones de docentes y directores de escuelas acerca delos factores que promueven u obstaculizan una educación ambiental compleja, generadora de conocimientos, con dispositivos participativos y orientados a la equidad en instituciones educativas del Área Metropolitana de Buenos Aires (Argentina). Como resultado se observaron experiencias heterogéneas y voluntad de instalar nuevas prácticas, pero con factores que limitan el cambio: pautas culturales arraigadas; deficiencias en la capacitación docente, en cuanto a las políticas públicas; poca claridad en las definiciones sobre los objetivos de la educación ambiental, su abordaje curricular y los dispositivos pedagógicos. Prevalecen enfoques fragmentados, naturalistas y la ausencia de una visión crítica. A nivel institucional, se discute la pertinencia de implementar prácticas sustentables en el aula. Las propias percepciones también operan como obstáculos. Todo esto dificulta la adopción de nuevas acciones de educación ambiental con el ímpetu necesario para sostenerlas en el tiempo.
\end{abstract}

Palabras clave: Percepciones. Docentes. Educación ambiental. Argentina.

\begin{abstract}
The paper examines the perceptions of teachers and directors regarding the factors that promote or constrain complex environmental education, which generates knowledge, with participatory devices and oriented to equity in educational institutions in Metropolitan Area of Buenos Aires (Argentina). As result, we detected heterogeneous experiences and willingness to set new practices up. But there are factors limiting the change: deep-rooted cultural customs; shortcomings in terms of teacher training; in public policies a lack of clarity about environmental education objectives, curricular approach and pedagogical devices. Fragmented and naturalists approaches, and lack of a critical perspective prevail. At the institutional level, the relevance of implementing sustainable practices in classroom is discussed. Perceptions also operate as obstacles. All this hinders the adoption of new environmental education actions and the momentum needed to sustain them over time.
\end{abstract}

Keywords: Perceptions. Teachers. Environmental education. Argentina.

\footnotetext{
${ }^{1}$ Universidad de Buenos Aires (UBA), Facultad de Ciencias Sociales, Buenos Aires, Argentina. E-mail:

$<$ msaidon@yahoo.com>.

${ }^{2}$ Universidad Nacional de Tres de Febrero, Buenos Aires, Argentina.
} 


\section{Introducción}

Existe un deterioro sustantivo en la calidad ambiental tanto a nivel global - primando en este plano el tema del cambio climático como problema fundamental - como en múltiples ámbitos locales (NACIONES UNIDAS, 2014). En términos generales, el rápido crecimiento poblacional y económico no fue acompañado por regulaciones y políticas ambientales acordes. Esto explica - en parte - la magnitud del impacto de la actividad antropogénica sobre lo ambiental. Ante ello, la Conferencia de las Naciones Unidas para el Medio Ambiente realizada en Estocolmo en 1972, incitó a los países allí participantes a implementar las medidas necesarias para revertir la tendencia del impacto antropogénico negativo sobre el ambiente. Como consecuencia, en esa época emergió con fuerza a nivel internacional la educación ambiental. En concreto, en cuanto a la educación, ese antecedente junto con las conferencias de Tibilisi (1977), Nairobi (1982) y Moscú (1987), sentaron las bases para la Conferencia de las Naciones Unidas para el Medio Ambiente y el Desarrollo de Río (1992) en la cual se acordó la Agenda 21, que estableció la adopción de un enfoque educativo basado en la concepción de "desarrollo sostenible" y el Tratado de Educación Ambiental. Luego, en el año 2000, en La Haya, se realizó una declaración internacional en la que estuvieron involucrados 46 países, incluyendo la participación de expertos y de organizaciones de la sociedad civil. Allí surgió la Carta de la Tierra, que expresa la necesidad de investigar las causas fundamentales de la crisis ambiental y las problemáticas ambientales y sociales que emergieron a partir del sistema económico vigente. Además, cuestiona la idea de orientar la educación ambiental hacia un desarrollo sostenible no interpelado, con la propuesta de priorizar el debate y una educación crítica. Finalmente, en el año 2003, por su parte, la Asamblea General de las Naciones Unidas declaró que se iniciaba la Década de las Naciones Unidas de la Educación para el Desarrollo Sostenible (Resolución A/RES/57/254) (NACIONES UNIDAS, 2002).

Tales encuentros y tratados internacionales, junto a la creación de jurisdicciones gubernamentales locales vinculadas a lo ambiental y al surgimiento de organizaciones no gubernamentales, dieron fuerza a la idea de introducir la educación ambiental en los planes de estudio y guiaron algunos emprendimientos en esta tarea. Además, promovieron que se incremente globalmente el nivel general de conocimientos con relación a lo ambiental en los últimos 50 años (FRANZEN, 2003). En consonancia, la educación ambiental comenzó a instalarse en las escuelas (GARCÍA COSTOYA; ZENOBI, 2009). Incluso, múltiples legislaciones a nivel nacional e internacional incorporaron la educación ambiental en el sistema de educación formal. Empero, aún existe cierto escepticismo respecto de la capacidad de los gestores para impulsar procesos educativos a favor de lo ambiental (CAMARENA GÓMEZ, 2006). La experiencia indica que a nivel internacional la escuela todavía no ha sido capaz de adecuar sus prácticas a los objetivos que se ha propuesto la educación ambiental (MEIRA CARTEA; CARIDE GÓMEZ, 2006). Diversos estudios muestran que los conocimientos básicos vinculados a los temas ambientales no llegan a convertirse en aprendizajes significativos en los alumnos (SAIDÓN, 2012).

\section{La educación ambiental en la Argentina: marco regulatorio, aplicación y estado del arte}

En particular en la Argentina, en los últimos años, la legislación se ha ido orientando a la promoción de la educación ambiental en general, tanto como en el sistema de educación 
formal. Como antecedente clave, en el año 2002 se aprobó la Ley General del Ambiente que, en su Artículo 8, enuncia que la educación ambiental es uno de los instrumentos de la Política y Gestión Ambiental. La misma Ley (en sus artículos 14 y 15), afirma que la educación ambiental constituye el instrumento básico para generar en los ciudadanos valores, comportamientos y actitudes que sean acordes con un ambiente equilibrado. Define a la educación ambiental como un proceso continuo y permanente, sometido a constante actualización, que deberá facilitar la percepción integral del ambiente y el desarrollo de una conciencia ambiental. También señala que las autoridades competentes deberán coordinar con los Consejos Federales de Medio Ambiente (COFEMA) y de Cultura y Educación, la implementación de planes y programas en los sistemas de educación formal y no formal. Finalmente, alude a la vinculación de la participación ciudadana en y mediante la educación ambiental afirmando que el/la ciudadano/a es el principal motor de cambio y de solución del conflicto ambiental (CONDENANZA; CORDERO, 2013).

Por su parte, la Ley de Educación Nacional, sancionada en el año 2006, fijó como uno de los objetivos de la política educativa argentina

[...] Brindar una formación ciudadana comprometida con los valores éticos y democráticos de participación, libertad, solidaridad, resolución pacífica de conflictos, respeto a los derechos humanos, responsabilidad, honestidad, valoración y preservación del patrimonio natural y cultural. (ARGENTINA, 2006, p. 2).

No obstante, no se han regulado lineamientos específicos de política educativa vinculados a la educación ambiental.

En tal sentido, y en relación con las experiencias educativas puestas en marcha hasta el momento, se advirtió que existen variados casos en cuanto a la generación de conocimientos vinculados a lo ambiental tanto a nivel internacional, como en los planos nacionales y locales. En la Argentina en particular, si bien se han establecido ciertos lineamientos generales en términos de educación ambiental para todas las instituciones, los Proyectos Educativos Institucionales (PEI) de las escuelas no siempre los incorporaron en sus propósitos didácticos. Al respecto, un estudio concluyó que en la Ciudad de Buenos Aires la educación ambiental en las escuelas primarias se aborda, en general, a través de actividades por fuera del currículum, dependiendo de la iniciativa particular de las instituciones y/o docentes. Este tipo de prácticas, si bien no garantiza que la educación ambiental se haga efectiva, genera una gama de propuestas heterogéneas y ricas para analizar y comparar en sus resultados (EDEL NAVARRO; RAMÍREZ GARRIDO, 2006; POTEL, 2012). Además, algunos estudios realizados en la Provincia de Buenos Aires concluyeron que un mayor nivel de educación formal no contribuye a garantizar - per se - la adquisición de conocimientos significativos básicos respecto de lo ambiental. Aún es necesario un trabajo de articulación transversal, discusión e incorporación de la educación ambiental en los diferentes niveles del sistema educativo y en los equipos encargados de los diseños curriculares, considerando que la educación ambiental pone en tensión la lógica disciplinar que históricamente asumió el currículo (BUENOS AIRES, 2007; SAIDÓN, 2012).

En cuanto al tema específico de las percepciones de los actores sobre lo ambiental en el sistema educativo, un trabajo realizado en Mendoza (Argentina) argumentó que los diferentes actores educativos poseen una concepción de ambiente acotada, biocéntrica y que abordan los problemas ambientales a escala planetaria (ERICE et al., 2010). En otro estudio se consultó 
a docentes de Geografía del nivel medio de la Ciudad de Buenos Aires sobre definiciones de lo ambiental, concluyendo que la educación ambiental se presenta como tema crecientemente requerido, demandado por parte de la comunidad educativa en general (FERNÁNDEZ CASO et al., 2010). Finalmente, un trabajo exploratorio realizado en escuelas de la Ciudad de Buenos Aires sobre las percepciones de los docentes sostuvo que si bien la educación ambiental se aplica en actividades escolares, enfrenta dificultades: las actividades se implementan de manera desarticulada y vía formatos extracurriculares, dependientes de la propia iniciativa de las escuelas y docentes. Al respecto, se subrayó la necesidad de introducir lo ambiental de manera más significativa en el diseño curricular. Asimismo, se destacaron dos puntos importantes: por un lado, una auto-percepción generalizada por parte de los docentes de deficiencias en conocimientos vinculados a lo ambiental; por otro, el rol de los medios de comunicación como fuente de información significativa de los docentes. Estos factores podrían estar confluyendo en que el abordaje del tema sea superficial y fragmentado (POTEL, 2012).

Algunos estudios desarrollados en el ámbito internacional, históricamente han venido señalando que la mayoría de los dispositivos de educación ambiental se han orientado a los problemas locales, sin abordar la globalidad (GONZÁLEZ GAUDIANO; ALBA CEBALLOS, 1994; HICKS; HOLDEN, 1995; TRAVÉ GONZÁLEZ; POZUELOS ESTRADA, 1999). También se ha hecho referencia a una percepción general respecto de un tratamiento reduccionista en las prácticas de educación ambiental, que suelen referir exclusivamente a los sistemas naturales sin prestar atención a las relaciones con el plano social (FIEN, 1995; TILBURY, 1995). Otras percepciones detectadas en los docentes se vincularon al abordaje superficial y fragmentado de lo ambiental (GIL PÉREZ; GAVIDIA CATALÁN; FURIÓ MÁS, 1997). En particular, un estudio realizado sobre docentes españoles subrayó que los mismos se sienten inseguros y manifiestan la necesidad de herramientas apropiadas: son pocos espacios escolares dedicados a la educación ambiental (BRAVO CHÁVEZ, 2011). No obstante, a pesar de estos aportes, un estudio realizado por Condenanza y Cordero (2013) señala que la investigación sobre educación ambiental en la Argentina es significativamente escasa, no habiéndose constituido como campo particular en la investigación educativa nacional.

Con todo esto, finalizada la Década de las Naciones Unidas de la Educación para el Desarrollo Sostenible, los resultados evidenciados en términos de la crisis ambiental vigente nos interpelan acerca de cuáles son los factores que inciden en los logros y fracasos en cuanto a los objetivos que se ha propuesto la educación ambiental. En primer lugar: ¿Es el "cuidado ambiental” orientado al Desarrollo Sostenible un concepto objetivo y/o neutral? ¿Qué objetivos debe proponerse la Educación Ambiental? Y sobre esa base: ¿Qué factores promueven una educación ambiental con ese perfil y cuáles la obstaculizan?

Considerando estos interrogantes, este trabajo, en primer lugar, propone una educación ambiental específica, orientada hacia ciertos objetivos concretos. Luego, examina de manera exploratoria cuáles son las percepciones que existen por parte de docentes y directores de instituciones educativas respecto de los factores que promueven u obstaculizan la adopción de prácticas de educación ambiental, orientadas a lograr aprendizajes significativos en los alumnos y la transferencia a sus entornos. Entendemos que las percepciones docentes son construcciones complejas, psicológicas, sociales, subjetivas, selectivas y temporales, elaboradas a partir de las propias experiencias personales y sociales. Para el problema educativo, estas percepciones se tornan altamente relevantes en cuanto a que el modo en que el docente interpreta los proble- 
mas ambientales son una causa necesaria de lo que los alumnos aprenderán (AUSUBEL, 2002). Para ello, se estudian perspectivas, posicionamientos, visiones, dilemas, prioridades, incentivos y concepciones, realizando un estudio cualitativo aplicado en los niveles de educación inicial, primaria y secundaria del Área Metropolitana de Buenos Aires (AMBA), Argentina. Los resultados permiten realizar aportes innovadores para corroborar explicaciones o predicciones académicas y lograr un mejor abordaje de las políticas públicas ambientales y educativas. Asimismo, sirven de base para delimitar y desarrollar nuevos estudios acerca de los alcances y desafíos de la educación ambiental en el siglo XXI.

\section{Marco teórico de referencia}

Para alguno autores, el desarrollo sostenible tiene un sentido claro (NACIONES UNIDAS, 2002) y, para otros, resulta un concepto vacío en sí mismo (UNMÜBIG; FATHEUER; SACHS, 2012), debiéndose hacer explícita la interpretación que cada quien da al referirse a tal concepto. En este trabajo se adopta un enfoque de desarrollo sostenible que, a la vez que busque garantizar un capital natural para las generaciones futuras, también promueva la justicia social. Debatir este punto excedería los objetivos de este trabajo. Asumimos como deseable una educación ambiental promotora de equidad inter-generacional e intra-generacional, considerando que los ciudadanos bien informados sobre los problemas ambientales son más conscientes de los posibles daños que podrían generar ciertas prácticas (DANIELSON et al., 1995).

Esta premisa nos permite afirmar que uno de los objetivos de la educación ambiental debe ser la generación de conocimientos. Pero, ¿Cómo debería abordarse el tema de los conocimientos ambientales? ¿Qué quiere decir comprender un fenómeno ambiental? Desde la psicología del aprendizaje existen diversas definiciones de conocimiento: las que enfatizan la habilidad para resolver lógicamente algún razonamiento, las que otorgan importancia a la capacidad para acumular información memorística, aquellas que subrayan la necesidad de un vínculo entre la temática en cuestión y su uso práctico (competencias), otras que enfatizan el desarrollo capacidad crítica, las que consideran indispensable la posibilidad de explicar un tema en los términos que lo hace la comunidad académica, etc. (POZO, 1997). En este trabajo, se adopta una concepción basada en una literatura que emergió en los 50s, cuando Bloom et al. (1956) desarrollaron el clásico trabajo Taxonomy of educational objectives: the classification of educational goals, que defiende una concepción de la dimensión cognitiva como la habilidadpara pensar cosas. Los autores propusieron distintos niveles cognitivos o de razonamientos: desde aquellos que son más básicos y relativamente concretos, hasta los más complejos y abstractos. Tales niveles fueron posteriormente ubicados en nuevas clasificaciones por diversos autores, destacándose Anderson y Krathwohl (2001); Fogarty y Stoehr (1995). Específicamente, en cuanto a los problemas ambientales, aquí asumimos que los mismos son sistémicos y que, por lo tanto, resulta pertinente evaluar los conocimientos en este campo en función de la capacidad de los individuos de construir estructuras conceptuales abstractas, que expliquen al ecosistema de manera integral, considerando al ambiente como sistema complejo en el sentido de Leff (1994), Morin (2001) y Sauvé (2005). En consonancia, en el ámbito de la educación ambiental, la North American Association for Environmental Education ha promovido una postura orientada a adherir a la concepción de generar y evaluar conocimientos vinculados a cómo pensar acerca del ambiente y no a quépensar sobre el mismo (HEIMLICH, 2010). 
En suma, a continuación definiremos dos grandes categorías de conocimientos ambientales que consideramos necesarios para lograr aprendizajes significativos desde nuestra perspectiva. La primera, se denomina Disponibilidad de Información. Este es un tipo de habilidad cognitiva que implica un conocimiento básico, aquel que (por sí mismo) no alcanza para una comprensión profunda, pero que se torna necesario y fundamental para el aprendizaje. La "información" incluye una acumulación de datos (ya sea conocimientos previos del alumno, como nuevos datos a incorporar) tales como nombres, hechos, rutinas, etc. que deben estar en concordancia con el conocimiento prevaleciente y dominante en las disciplinas que abordan la temática ambiental (la química, la biología, la ingeniería, etc.). Esta categoría es consistente con la clase de conocimiento a la que Fogarty y Stoehr (1995) denomina factual thinking/gather o Ausubel (2002) subsunsor. También podría asociarse a la categoría remember de Anderson y Krathwohl (2001) y con el nivel más simple de conocimiento propuesto por Bloom et al. (1956). La segunda categoría es la de comprensión. Esta dimensión de conocimiento implica la capacidad de aplicar conceptos, interpretar, realizar inferencias, integrar el conocimiento y organizar la información. Significa haber trascendido la memorización de la información, la intuición y la reproducción de conocimientos básicos. En consecuencia, la información disponible puede utilizarse para construir un conocimiento más amplio y complejo. Esto puede incluir procesos de razonamiento tales como analizar las ventajas y desventajas de ciertos mecanismos o realizar un análisis de factibilidad. La categoría de la comprensión está en línea con Boix Mansilla y Gardner (2005), quienes la interpretan como la habilidad de utilizar el conocimiento en situaciones novedosas mediante la conformación de una red conceptual, organizada en forma consistenten - con ejemplos y generalizaciones - y en correspondencia con los criterios comúnmente aceptados en la/s disciplina/s de origen. Asimismo, implica una capacidad de contemplar el contexto socio-económico y político, y una construcción del conocimiento que problematice las situaciones del contexto, con un pensamiento crítico. Esta categoría es consistente con la idea de aprendizaje significativo mencionada anteriormente o equilibración de Piaget (1978). También incluye los dos niveles superiores de conocimiento propuestos por Fogarty y Stoehr (1995): critical thinking/process y creative thinking/ apply, sintentiza los niveles de conocimiento understand, apply, analyze, evaluate y create presentados en el trabajo de Anderson y Krathwohl (2001) y cubre los niveles más complejos propuestos por Bloom et al. (1956): evaluation, comprensión, synthesis, analysis y application.

Ambas categorías, además, pueden ponerse en diálogo y son consistentes con el enfoque del modelo pedagógico constructivista, en el cual un proceso de enseñanza que se asume como rico es aquel que permite dotar a los alumnos de información y herramientas para construir sus propios procedimientos para resolver problemas. Es en la interacción entre la información (desequilibrio) y esquemas de pensamiento, que se construye y complejiza el conocimiento.

A su vez, se asume como deseable un proceso de enseñanza dinámico y participativo, orientado a la acción, en el sentido de Piaget $(1980,1983)$ y en el de quienes estudiaron y desarrollaron sus ideas posteriormente. También, siguiendo a Freire (1998), se busca una educación que estimule la autonomía del pensamiento, basada en una relación educativa que, aunque asimétrica, sea dinámica y dialéctica. En suma, la investigación adscribe - y desde allí se pregunta y reflexiona - a una educación ambiental que involucra procesos participativos, de deliberación y toma de decisiones por parte del alumno. Este modelo, a su vez, favorece la formación ciudadana (GUREVICH, 2011) y promueve la crítica y la construcción de un pensamiento complejo, que articula relaciones sociales y naturales (RIVAROSA et al., 2010). 
Por otro lado, en cuanto al aspecto de las instituciones, siguiendo a North (1990) planteamos que los límites para la implementación de prácticas de educación ambiental en los establecimientos educativos podrían asociarse a que las instituciones suelen tener un desenvolvimiento atado al pasado, con cierta resistencia al cambio. En consonancia con esta perspectiva, las prácticas asociadas a la educación ambiental pueden estar mediatizadas por intereses divergentes, por recursos diversos, por agentes heterogéneos y por factores políticos, sociales y económicos (GUTIÉRREZ PÉREZ; POZO LLORENTE, 2006) que generarían una dependencia respecto de trayectorias pasadas.

Tales premisas teóricas orientaron las siguientes preguntas: conociendo la problemática ambiental, ¿a qué se debe que muchas instituciones no hayan realizado aún actividades de educación ambiental promotoras de una mayor apropiación de conocimientos por parte de los alumnos? A su vez, cuando tales prácticas se hubieran efectuado, ¿han logrado promover una comprensión profunda del tema, más allá de la transmisión de información básica? ¿Han conseguido la participación de los alumnos, la construcción de procedimientos propios para resolver problemas y tomar decisiones? ¿Por qué? ¿Por qué algunas experiencias educativas se inician y no permanecen en el tiempo? ¿Cómo lo perciben docentes y directivos? ¿Cómo perciben docentes y directivos los factores que promoverian u obstaculizarian la implementación de prácticas de educación ambiental? Este trabajo propone un abordaje de tipo exploratorio para comenzar a dar respuestas a estas preguntas y viabilizar futuros estudios que las sigan trabajando.

\section{Marco metodológico}

Esta investigación se centró en el estudio de percepciones de directivos y docentes de instituciones educativas. Para ello, se apeló a la consulta de fuentes de información secundaria como documentos institucionales, así como a material académico teórico y aplicado. A su vez, se trabajó con fuentes de información primaria, con un total de 21 casos sobre los cuales se realizaron registros. En particular, se implementaron entrevistas, observaciones participantes y registros de discursos de directores y docentes del sistema formal en el AMBA. Esta región, que agrupa a la Ciudad de Buenos Aires y las localidades linderas de la Provincia de Buenos Aires, posee en su conjunto características muy disímiles, ya que se compone por zonas más o menos residenciales y de clases sociales estratificadas. Esta Área se constituye como el sector con mayor población y uno de los polos industriales y económicos más dinámicos del país.

Las escuelas sobre las que se realizaron registros corresponden a nivel inicial, primario y secundario. Lo datos se tomaron en el marco de la realización de 6 entrevistas, así como en charlas y talleres de capacitación sobre temas de educación ambiental. Se trata de un trabajo realizado a lo largo de tres años, en donde se registraron comentarios y discusiones en diversos eventos y jornadas de trabajo, con docentes y directivos en distintos ámbitos: grupos de formación docente en universidades; talleres de capacitación docente en escuelas; y ateneos de investigación en educación ambiental realizados por equipos de investigación. Las observaciones de las prácticas estuvieron guiadas por las preguntas de investigación señaladas previamente. En particular, se observó la existencia o no de prácticas sobre lo ambiental en las escuelas y sus características. También, se buscó conocer las percepciones de docentes y directivos; se observaron las perspectivas, posicionamientos, prioridades e incentivos de los principales actores involucrados en la educación ambiental en la escuela. Específicamente, se trabajó con casos 
de docentes y directivos que cumplen tareas en instituciones (públicas y privadas) pertenecientes a tres municipios: dos distritos de la Provincia de Buenos Aires (Quilmes y San Isidro) y la Ciudad Autónoma de Buenos Aires. Como se trata de una investigación exploratoria, los casos tomados para el análisis no fueron previstos con un criterio distributivo específico. No obstante, dadas las características de los distritos considerados y la ubicación geográfica de las instituciones seleccionadas, los casos tomados abarcaron sectores socio-económicos heterogéneos. El carácter exploratorio de la investigación permitió manejar el análisis con categorías abiertas y flexibles, dando lugar a que pudieran emerger factores que resultaran novedosos. Por tal sentido, se realizaron entrevistas semiestructuradas, incluyendo preguntas abiertas. Luego, el trabajo sobre la información primaria y secundaria disponible, nos permitió elaborar el análisis con los resultados que se presentan en el próximo apartado a partir de la propuesta de agrupamientos y clasificaciones sobre las percepciones registradas. Por las características de la muestra, la distinción entre sector público y privado no fue un criterio considerado significativo y -por lo tanto- válido para la construcción de las dimensiones, con lo cual esta comparación no adquirió un carácter relevante en las conclusiones del estudio. A partir del análisis de los datos han quedado constituidos las dimensiones e indicadores que se detallan en el Cuadro 1.

Posteriormente, se desarrollan las conclusiones y reflexiones derivadas del análisis de las entrevistas bajo las dimensiones señaladas.

\section{Resultados: análisis de experiencias y percepciones}

En esta sección se analizan las características generales de las prácticas y concepciones en el campo de la educación formal evidenciadas a través de las fuentes de información disponibles.

En primer lugar, sobre la base de los registros tomados, se detectaron instituciones con presencia de experiencias de educación ambiental. Sin embargo, el alcance de estas experiencias difiere respecto del nivel de cobertura que se abarca en cada una con relación al alumnado. A su vez, las experiencias educativas existentes se presentan como disímiles en cuanto a la generación de conocimientos ambientales, en vinculación a lo que domina en las disciplinas de base. En general, no aparece la idea de complejidad (en términos de redes y vínculos entre naturaleza y sociedad, así como al interior de ambas categorías) y el abordaje de lo ambiental no suele ser integral. Este hecho coincide con estudios que argumentan que lo ambiental suele estudiarse de manera fragmentada (CORBETTA; SESSANO; KRASMANSKI, 2012).

Por otra parte, si bien a nivel nacional, provincial y distrital se fijaron ciertos lineamientos generales en términos de educación ambiental para todas las instituciones; los PEI de los distintos establecimientos -abarcando la contextualización del diseño curricular, los criterios metodológicos, los materiales utilizados, etc.- varían en las formas de su incorporación. Esta heterogeneidad responde a distintas cuestiones. Por ejemplo, contar (o no) con incentivos del sector público, con la cooperación de organizaciones de la sociedad civil o bien con el aporte de padres y/o diversos actores que se acercan con propuestas específicas. También se vincula con demandas diferentes por parte de los alumnos, que están insertos en contextos específicos y expuestos a noticias de los medios de comunicación que parecen incidir de manera desigual (QUINTERO; SAIDÓN; BACHMAN, 2014). 
Cuadro 1. Dimensiones e indicadores de análisis

\begin{tabular}{|c|c|c|c|}
\hline Etapas & Dimensión & Indicadores & Preguntas/Temas de interés \\
\hline $\begin{array}{c}\text { Primera } \\
\text { observación }\end{array}$ & $\begin{array}{l}\text { Existencia (o no) } \\
\text { de experiencias } \\
\text { de educación } \\
\text { ambiental en la } \\
\text { escuela y carácter }\end{array}$ & $\begin{array}{l}\text { Existencia } \\
\text { Nivel de cobertura } \\
\text { Complejidad } \\
\text { Condiciones }\end{array}$ & $\begin{array}{l}\text { ¿Hay educación ambiental en la escuela? } \\
\text { ¿En qué marco se da? } \\
\text { ¿Con qué complejidad se aborda? } \\
\text { ¿Cuáles son las condiciones de las prácticas } \\
\text { educativas? } \\
\text { ¿Qué proporción abarca del alumnado? }\end{array}$ \\
\hline \multirow[t]{5}{*}{$\begin{array}{l}\text { Clasificación } \\
\text { de las } \\
\text { percepciones } \\
\text { de los } \\
\text { docentes y } \\
\text { directivos }\end{array}$} & 1. Responsabilidad & $\begin{array}{l}\text { Instancias de } \\
\text { responsabilidad } \\
\text { Omisiones } \\
\text { Acciones }\end{array}$ & $\begin{array}{l}\text { ¿Cuál consideran que es el grado de } \\
\text { responsabilidad de los diversos actores por } \\
\text { las problemáticas ambientales existentes? } \\
\text { ¿Cuán responsable es la escuela? } \\
\text { ¿Cuál es el rol de la escuela al respecto? }\end{array}$ \\
\hline & 2. Abordaje & $\begin{array}{l}\text { Tipos de abordajes } \\
\text { teóricos } \\
\text { Dispositivos } \\
\text { institucionales (PEI) } \\
\text { Definiciones y } \\
\text { objetivos de lo } \\
\text { ambiental }\end{array}$ & $\begin{array}{l}\text { ¿Cuáles son las concepciones teóricas sobre } \\
\text { el aprendizaje escolar y lo ambiental en la } \\
\text { institución? ¿Hay definiciones y objetivos } \\
\text { institucionales sobre lo ambiental? } \\
\text { ¿Qué visiones se enfrentan y qué } \\
\text { preguntas surgen en cuanto al abordaje? } \\
\text { ¿Se corresponden con las discusiones } \\
\text { académicas en cuanto al abordaje? } \\
\text { ¿Cuáles son los dispositivos de enseñanza } \\
\text { de lo ambiental? ¿Se trabaja tanto la } \\
\text { dimensión natural como la social? ¿Existe } \\
\text { abordaje diferenciado por grupos etarios? }\end{array}$ \\
\hline & $\begin{array}{l}\text { 3. Gestión } \\
\text { ambiental en la } \\
\text { escuela }\end{array}$ & $\begin{array}{l}\text { Prácticas } \\
\text { institucionales } \\
\text { Vínculo con la } \\
\text { comunidad local }\end{array}$ & $\begin{array}{l}\text { ¿Hay prácticas sustentables en la escuela? } \\
¿ \text { ¿ómo se maneja la dualidad discurso- } \\
\text { gestión? ¿Qué posibilidades educativas } \\
\text { brinda la gestión? } \\
\text { ¿Hay interacción con la comunidad local? }\end{array}$ \\
\hline & $\begin{array}{l}\text { 4. Formación } \\
\text { docente }\end{array}$ & $\begin{array}{l}\text { Saberes docentes en } \\
\text { la temática ambiental } \\
\text { Articulación } \\
\text { transversal y } \\
\text { longitudinal de } \\
\text { contenidos } \\
\text { Dispositivos } \\
\text { pedagógicos } \\
\text { Currículum }\end{array}$ & $\begin{array}{l}\text { ¿Cuáles son los saberes docentes respecto } \\
\text { de lo ambiental? } \\
\text { ¿Cuáles son las fuentes de conocimiento } \\
\text { docente? }\end{array}$ \\
\hline & $\begin{array}{l}\text { 5. Importancia de } \\
\text { lo ambiental en la } \\
\text { escuela }\end{array}$ & $\begin{array}{l}\text { Importancia sobre } \\
\text { "otros temas" } \\
\text { Agenda educativa }\end{array}$ & $\begin{array}{l}\text { ¿Existe capacidad de articular los } \\
\text { contenidos en las clases, entre materias y } \\
\text { años escolares? } \\
\text { ¿Se incorporan contenidos específicos? } \\
\text { ¿Cuál es el nivel de actualización docente? } \\
\text { ¿Qué importancia asigna la escuela al } \\
\text { problema del ambiente? } \\
\text { ¿Está instalado el debate en la agenda } \\
\text { educativa? }\end{array}$ \\
\hline
\end{tabular}

Fuente: Elaborado por las autoras. 
A la vez, por propia iniciativa de docentes o directores, algunas instituciones educativas suelen proponer la realización de este tipo de actividades educativas sobre diversas cuestiones vinculadas a lo ambiental (FERNÁNDEZ CASO et al., 2010). Por ejemplo, sobre el uso racional de la energía, hidrocarburos, reciclado de residuos, cuidado del ambiente en general, etc.

Asimismo, en gran parte de los establecimientos se manifiesta la voluntad de adoptar prácticas ambientalmente sostenibles al interior de las instituciones, así como de realizar actividades de educación ambiental. No obstante, se advierte una resistencia al cambio. En particular, existen algunas percepciones que refieren a factores promotores del cambio, así como a otros obstaculizadores. A continuación, se analizan los resultados obtenidos, clasificando tales percepciones de docentes y directivos en distintas dimensiones.

\section{La responsabilidad}

Algunos docentes y directores manifiestan que la problemática ambiental actual involucra en gran medida a corporaciones, gobiernos, etc. y que, por lo tanto, la responsabilidad de involucrarse en este tema no se puede adjudicar a la comunidad local y, menos aún, a la escuela. En el mismo sentido, hay quienes aluden a deficiencias en las políticas públicas de los distintos niveles de gobierno. En consecuencia, plantean la dificultad de adoptar nuevas prácticas educativas sin un acompañamiento acorde con las políticas que se implementan.

En contraposición, muchos apoyan la idea de que la escuela debe involucrarse como responsable frente a lo ambiental, expresando la necesidad de reflexionar en el aula sobre el tema: justamente, se sostiene, podría ponerse en discusión la cuestión de las responsabilidades relativas respecto de la crisis ambiental vigente. Así, en línea con la Carta de la Tierra, se cuestiona la idea de orientar la educación ambiental hacia un desarrollo sostenible no problematizado. En este sentido, se oyen voces que argumentan que no debemos quedar paralizados ante los grandes poderes. El hacer caso omiso a lo que ocurre, bajo el argumento de la ausencia de responsabilidades, sería un comportamiento reaccionario, en el sentido de Freire (1998). Es decir, naturalizar el estado de situación a nivel planetario y asumir que el educando se debe adaptar a la realidad y que nada puede hacer para alterarla. En cambio, puede proponerse una educación "problematizadora" y no "naturalizadora".

Además, también en defensa de instalar la educación ambiental en la escuela, hay quienes sostienen que todos tenemos cierta parte de responsabilidad respecto de la sostenibilidad del sistema en el que nos desenvolvemos. En este sentido, se plantea que la comunidad tiene responsabilidad - como consumidora, como generadora de residuos, etc. - y que, en este campo, también debe intervenir la escuela como institución perteneciente a esa comunidad. En cuanto al manejo de residuos, por ejemplo: ¿estamos ahorrando papel al imprimir en la escuela?, cuando trabajamos respecto de la descomposición de los productos orgánicos ¿analizamos qué se está haciendo con todos los residuos orgánicos que produce nuestra escuela y qué se podría hacer? Cuando hablamos de los "bichos", ¿mencionamos su rol como descomponedores de la materia orgánica? Así, se considera que los centros educativos tienen especial relevancia, justamente, por su función educadora. 


\section{El abordaje}

La falta de claridad respecto del abordaje a adoptar frente al tratamiento de lo ambiental en la escuela muchas veces resulta paralizadora para instalar iniciativas educativas. En la mayoría de las instituciones se sostiene que aún deben definirse objetivos en torno a la educación ambiental. Algunos subrayan la necesidad de formar alumnos capaces de ser autónomos y con capacidad de creación (EDEL NAVARRO; RAMÍREZ GARRIDO, 2006). Hay quienes sostienen la necesidad de una práctica ambiental crítica y transformadora (GARCÍA COSTOYA; ZENOBI, 2009). Otros, enfatizan la necesidad de instalar el conflicto, en lugar de dar soluciones. Algunos también hablan de la necesidad de visibilizar procesos subyacentes. Otros, de lograr un análisis complejo de lo ambiental (LEFF, 1994; MORIN, 2001; SAUVÉ, 2005).

Si bien inicialmente las escuelas abordaban la educación ambiental como asociada a temáticas principalmente ecológicas, recién alrededor de los años noventa las cuestiones ambientales comenzaron a incorporar las ciencias sociales, ampliando el enfoque que las restringía al campo de las ciencias naturales. Este cambio permite considerar una perspectiva social y crítica de la educación ambiental (GARCÍA COSTOYA; ZENOBI, 2009). Sin embargo, si bien esa parece ser la tendencia, en las entrevistas realizadas el trabajo de lo ambiental aún no emerge como un contenido (también) social que podría cruzar transversalmente todas las materias articulando temáticas y conocimientos. Por otra parte, algunos plantean dudas respecto de cómo sostener un discurso acerca de comportamientos "amigables" con lo ambiental, cuando la escuela no puede responder a eso con sus prácticas (por ejemplo, reduciendo el consumo de material descartable o con el reciclaje de los residuos que produce). Una respuesta que ha resultado útil al respecto para alguna institución es trabajar el tema de la tolerancia: tolerar la espera, prioridades y tiempos del otro.

En otro plano, hay quienes se preguntan acerca de la pertinencia de formar en valores sobre lo ambiental. Si bien unos consideran excesivamente moralizador y/o verticalista la transmisión de valores, otros proponen perder el miedo a la enseñanza ética y que, así como se realiza en otros ámbitos - el de la educación sexual, el de los derechos humanos, etc. -, también los valores ambientales deben transmitirse en la escuela (EDEL NAVARRO; RAMÍREZ GARRIDO, 2006). Hay quienes plantean que los alumnos son "futuros adultos" y que, por lo tanto, debemos enseñarles a desnaturalizar el presente para preservar ese futuro. Sin embargo, otros alegan que no debemos generar angustia y proponen, en cambio, pensar en un enfoque alternativo. Por ejemplo, abordar el trabajo desde la belleza de la naturaleza. Entonces, ¿Debemos pensar la educación ambiental desde una perspectiva fatalista, desde una optimista o de qué manera y en qué casos?

A su vez, se advierte una concepción bastante generalizada respecto de que es necesario diferenciar, en educación ambiental por grupos etarios, así como se hace en otros campos de la enseñanza. Sin embargo, en el campo de lo ambiental, esto adquiere especial relevancia, considerando la capacidad de los distintos grupos de asimilar las características y posibles consecuencias crecientemente críticas de la crisis ambiental a la que se enfrenta el planeta en general y ciertas áreas locales, en particular. En este sentido, los niveles de responsabilidad por las acciones individuales son distintos según la edad alcanzada. También la capacidad de abstracción, de acuerdo con la edad, incide en las posibilidades de adquirir mecanismos complejos de razonamiento. En los grupos de primera infancia se argumentó que conviene trabajar estos 
temas con un abordaje que parte de lo concreto y estudia las propias prácticas, más que la teoría. En cuanto a las capacidades de asimilación y acomodación, en algunos casos existe un posicionamiento respecto de que estos temas deben abordarse en función de las preguntas que vayan surgiendo y no más allá de ello. Además, se manifestó la necesidad de trabajar las problemáticas ambientales significativas evitando los fatalismos, sobre todo, en grupos de menor edad.

También resultan recurrentes otros interrogantes concretos que divergen en cuanto al abordaje: ¿Existen métodos universales?, ¿Deben ser necesariamente vivenciales los dispositivos? $¿$ Se requiere indispensablemente de un trabajo apoyado en salidas de campo? ¿Es necesaria la interacción con la comunidad? ¿Cuánto pueden aportar los medios de comunicación en los dispositivos de enseñanza-investigación? ¿Debe eliminarse el formato de bajada de conocimientos desde el docente hacia el alumno? ¿Qué hacer cuando existe desconocimiento generalizado por parte de los alumnos respecto de cuestiones técnicas y discusiones académicas relevantes que emergen en las distintas disciplinas?

En consecuencia, se observan múltiples interrogantes, así como diferencias significativas en las concepciones y estrategias de educación ambiental. Algunas más naturalistas, otras con componentes sociales. Algunas más vivenciales, otras más teóricas. Unas más naturalizadoras del modelo vigente y otras más críticas. Algunas más doctrinarias y dogmáticas; otras más participativas.

Finalmente, las diferencias relativas a distintos enfoques que emergen típicamente en el ámbito académico (como podría ser el debate entre un abordaje preservacionista -versus uno conservacionista- u optar por un enfoque naturalista, científico, humanista, holístico, de la sostenibilidad, de eco-educación, etc.) no surgen como preocupación relevante en el ámbito de las escuelas, si bien apareció en algún caso aislado. Esto podría dar cuenta de cierta lejanía entre la formación docente y los temas que se discuten en las disciplinas académicas asociadas al ambiente.

\section{La gestión ambiental: ¿en la escuela?}

Con respecto a la pertinencia de realizar una gestión de lo ambiental en la escuela realizar acciones cotidianas consistentes con los valores ambientales que sostienen docentes y directivos- se han evidenciado también distintos posicionamientos.

Por una parte, hay quienes sostienen que la escuela no debe sumar la responsabilidad de "gestionar lo ambiental", sino, solo cumplir con su función educadora a través de las didácticas que se desarrollen en el aula. En contraposición, otra postura alega que la escuela puede realizar una gestión ambiental adecuada - actuando localmente - y tiene una responsabilidad de ejercer en la práctica lo que aboga en su discurso, adoptando una posición activa en lo que se vincula a su impacto sobre el ambiente. Por ejemplo, de acuerdo con un testimonio: "[...] la escuela no puede predicar una cosa y hacer otra (no separar, entregar materiales con excesivo uso de papel, derrochar energía dejando las luces encendidas cuando no bace falta, etc.)”. Muchas veces, incluso, también se propone que la escuela gestione proyectos ambientales que la excedan: en el ámbito del barrio o de la comunidad local. Asimismo, se plantea que las posibilidades educativas de vivenciar ciertas prácticas son enormes y que la gestión permite debatir y promover decisiones participativas y bajo la adquisición de conocimientos diversos. Esto es consistente con el argumento que sostiene que la escuela debe asumir responsabilidades de manera creativa, generar una educación integral en 
donde los alumnos no solo sean receptores de información, sino que sean educados para la vida mediante un trabajo participativo y formador de criterios, estando directamente vinculados a la realidad ambiental (EDEL NAVARRO; RAMÍREZ GARRIDO, 2006).

\section{La formación docente}

Un tema recurrente en los argumentos que recorren las dificultades que enfrenta la escuela a la hora de emprender actividades de educación ambiental es el de las falencias en cuanto a formación docente. Esto hace que muchos educadores, según lo explican, realicen prácticas sobre la base de su intuición y/o de lo que toman directamente de los medios de comunicación, por ejemplo. Sin embargo, a la hora de problematizar y complejizar el abordaje de lo ambiental, así como de trabajarlo de manera integral, esto resulta insuficiente. Por ejemplo, directores entrevistados sostienen que, si bien existe en muchos casos una buena predisposición a trabajar la temática ambiental, se advierte una dispersión muy grande entre los conocimientos disponibles por los distintos docentes y que estos son escasos muchas veces. Sobre todo, si se está pensando en que los docentes puedan vincular la temática ambiental, de manera permanente, con otros contenidos curriculares.

Además, si bien existen algunas instancias de formación docente en educación ambiental, las mismas no están extendidas a todo el sistema de formación.

En consonancia, diversos estudios a nivel nacional señalaron grandes deficiencias en cuanto a formación docente en educación ambiental (CHROBAK et al., 2006). En el mismo sentido, distintos trabajos expresan que existe cierta base de conocimientos en los docentes que podría ser útil para abordar la cuestión ambiental, pero que la misma suele ser diferente entre ellos (FARROKHIAN; HOSSEINPOUR; SOLEIMANI, 2012). Al respecto, tanto en cuanto a las diferencias, como en cuanto a las deficiencias, se recomienda que los docentes que implementen educación ambiental reciban capacitaciones que los expongan a una formación integral y a los nuevos temas que van emergiendo. Se destaca que, más allá de la intuición y de la información provista por los medios de comunicación -lo cual genera conocimientos frecuentemente fragmentados- es necesario capacitar docentes desde la formación básica y a través de otros cursos posteriores complementarios (POTEL, 2012) orientados a las formación y actualización.

\section{La importancia de lo ambiental por sobre otros temas de la escuela}

En las entrevistas realizadas, algunos explican que la cantidad de temas a los cuales "debería abocarse la escuela" suele exceder sus posibilidades. En este sentido, se argumenta que lo ambiental es un tema más y que la escuela debe priorizar algunos temas por sobre otros: parece que los directivos se sienten "tironeados" por múltiples actores que demandan excesivamente a la escuela desde distintos ámbitos y disciplinas. Además, algunos remarcan que existen otras obligaciones y actividades curriculares que se perciben como prioritarias al interior de los establecimientos educativos y relegan entonces, frecuentemente, la instalación de nuevos dispositivos de educación ambiental. Diversos docentes y directores argumentan que la educación ambiental implica mayores costos en términos de tiempos y de recursos humanos, principalmente. Se arguye que la educación ambiental desarticularía la estructura escolar. 
Sin embargo, en otro sentido, hay quienes explican que la educación ambiental ha resultado rica, en cuanto a que ha posibilitado abordar algunos problemas irresueltos o cuestiones complejas de discutir en la escuela. A través de lo ambiental puede trabajarse, por ejemplo, el tema de la ética y la política (GUREVICH, 2011), el de la violencia y los límites, el de la solidaridad, contribuir al desarrollo de una visión crítica, a estimular la creatividad, la participación, etc. En consonancia, también se alega que lo ambiental puede instrumentarse como un tema transversal a todo lo cotidiano y a los diversos contenidos curriculares y que no requiere de recursos y tiempos adicionales. Se trata de repensar las actividades de la escuela en todos los ámbitos. De una mayor calidad educativa y de una mejor gestión de lo educativo.

\section{Otras cuestiones}

Más allá de todos estos puntos, han surgido otros temas sobre los que también valdría la pena seguir profundizando: ¿Es cada comunidad educativa la que debe priorizar y definir su propia agenda ambiental o existen temas prioritarios o recomendables sobre los que trabajar? Tampoco existe una percepción y un posicionamiento claro entre actores respecto de si la educación ambiental debe responder a lineamientos que bajen jerárquicamente desde las instituciones gubernamentales hacia las educativas, o bien, si estos deben ser definidos por cada establecimiento (en función del contexto ambiental en el que cada uno se inserta, de las inquietudes de los alumnos, etc.).

Otra preocupación se vincula a cómo repercute la educación ambiental sobre el vínculo de los alumnos con el afuera de la comunidad educativa. Algunos docentes explican que los chicos, cuando salen no pueden aplicar lo aprendido: "¿De qué sirve enseñarles a separar residuos adentro, si en sus casas no pueden hacerlo?". Sin embargo, otros sostienen que "[...] algunas veces los alumnos nos sorprenden: una alumna generó un sistema de separación de residuos en un barrio cerrado, por ejemplo[...]”.

\section{Reflexiones finales}

La educación formal se presenta como una de las vías propuestas por la comunidad internacional para generar conciencia y cambios actitudinales en un terreno complejo como el ambiental, en el cual intervienen actores con capacidades e intereses diversos. Hemos planteado un enfoque teórico que considera que una comprensión profunda brinda a los alumnos las habilidades para pensar y hacer en consecuencia. Es decir, no alcanza la transmisión escolar de una cuota de información sobre los problemas del ambiente, si eso no se ve acompañado por nuevas prácticas escolares más sustentables, una articulación transversal de los contenidos y una propuesta de pensar el problema de lo ambiental de forma compleja, considerando, por ejemplo, las ventajas y desventajas de ciertos escenarios naturales y sociales. En este sentido, la educación ambiental emerge como una herramienta interesante para poner en práctica modalidades participativas de adopción de decisiones - de construcción de ciudadanía-, para fomentar una educación crítica, para abordar los conocimientos desde un enfoque complejo y en permanente vinculación con los temas que trabajan las múltiples disciplinas científicas asociadas a lo ambiental.

Este trabajo se propuso identificar percepciones de docentes y directivos en relación a este tema, con un carácter exploratorio, a fin de abrir nuevos interrogantes para pensar líneas 
de acción futuras en el marco de la investigación académica y la política educativa, en un campo que aún no ha sido estudiando con profundidad en el país. Para ello, clasificamos la información proveniente de observaciones y entrevistas abiertas, en cinco dimensiones de análisis que nos permiten una aproximación al contexto del problema de la educación ambiental en el Área Metropolitana de Buenos Aires. Concluimos que las prácticas de educación ambiental en el sistema de educación formal no están plenamente instaladas en las escuelas. Incluso, si bien en algunas instituciones aparecen ciertas actividades o contenidos concretos, esto no significa que las mismas alcancen a todos los alumnos de la institución. Observamos que cuando tales experiencias ocurren, la calidad educativa no está garantizada. Muchas veces, rige un abordaje fragmentado de la realidad ambiental e, incluso, una visión naturalista (sin un tratamiento relativo de la dimensión social). Algunas veces, también se advierten falencias en cuanto a la capacidad de desarrollo de una visión crítica por parte de los alumnos. Además, con frecuencia, los contenidos de educación ambiental no consideran los conocimientos y discusiones vigentes y dominantes en las disciplinas científicas que trabajan el tema.

Por otra parte, se advirtió que la educación ambiental es bastante heterogénea entre las instituciones. Las experiencias educativas detectadas se presentan como sustantivamente disímiles en cuanto al trabajo de temáticas específicas, de abordajes disciplinares concretos, en términos metodológicos y de formación docente. Esta heterogeneidad puede atribuirse a distintas cuestiones que incentivan o paralizan el trabajo de temáticas ambientales en el aula, o bien, motivan la emergencia de distintos formatos. Tal heterogeneidad, a su vez, por una parte, impide garantizar cierta base de conocimientos comunes. Sin embargo, en contraposición, genera una diversidad de experiencias educativas que resulta rica, en tanto involucra y admite distintas percepciones asociadas a contextos específicos. Se observó la existencia de experiencias variadas que responden a construcciones conjuntas entre docentes y alumnos, de acuerdo con sus intereses y demandas concretas.

De acuerdo con la percepción de docentes y directores existen pautas culturales arraigadas (institucionales y sociales) que limitan, en cierto sentido, la instalación de nuevos temas y dispositivos. Sin embargo, se advierte una expresión bastante generalizada de voluntad de realizar actividades de educación ambiental por parte de los mismos.

Como obstáculos, se identificó que opera la falta de claridad respecto al abordaje que debe adoptar la educación ambiental, a los objetivos, al nivel jerárquico en el que estos deberían determinarse, a los tiempos y recursos que esto implicaría, deficiencias en términos de capacitación docente, la inercia institucional y una percepción compartida respecto de falencias en las políticas públicas vinculadas a lo ambiental. Esto debería atenderse y discutirse en instancias políticas, académicas y en conjunto con la comunidad educativa. También genera cierta resistencia a la puesta en marcha de actividades de educación ambiental, la falta de definiciones respecto del rol de la escuela como educadora y gestora de lo ambiental, ante la percepción de que existen (otros) grandes actores con mayor responsabilidad por la crisis ambiental vigente y otros temas que se proponen como prioritarios a ser trabajados.

Por su parte, si bien hay quienes cuestionan la idea de gestionar (de generar prácticas concretas) lo ambiental en las instituciones educativas, de acuerdo con otras visiones, la escuela debe ser responsable por debatir acerca de lo ambiental, de gestionar lo ambiental-para garantizar la consistencia con su discurso- y debe plasmar la educación ambiental (de manera transversal) en todos sus ámbitos. En este sentido, consistentemente con el enfoque teórico aquí propuesto, se plantea que la incorporación de la gestión en la educación es indispensable para que los 
alumnos se apropien del conocimiento mediante dispositivos participativos, vivenciales y, a su vez, comprometidos con la comunidad, adhiriendo a una postura orientada a la concepción de generar y evaluar conocimientos vinculados a cómo pensar acerca del ambiente y no a quépensar sobre el mismo. También merece la atención cierta resistencia a la idea de orientar la educación ambiental hacia un desarrollo sostenible no interpelado y se propone priorizar el debate al respecto.

Este tipo de dilema, para muchos no resuelto, dificulta la posibilidad de adoptar nuevas prácticas de educación ambiental con el ímpetu necesario para sostenerlas en el tiempo. En este sentido, una conclusión novedosa tiene que ver con que las propias percepciones operan en muchos casos como obstáculos o factores promotores para la implementación de prácticas de educación ambiental en la escuela. Avanzar sobre estas discusiones, entonces, podría contribuir a afianzar nuevos modelos de educación ambiental. Estos debates abiertos en sí mismos pueden resultar enriquecedores para el desarrollo de prácticas escolares de educación ambiental.

Estos resultados, además, son consistentes con los estudios de antecedentes en el plano nacional e internacional, en cuanto corroboran un abordaje fragmentado del problema de lo ambiental en las escuelas y escasa formación docente para encarar nuevas prácticas educativas. Proponemos retomar las conclusiones de este trabajo exploratorio, en nuevas investigaciones que analicen y discutan las dimensiones construidas: ¿existe educación ambiental en las escuelas de la Argentina? En caso afirmativo: ¿quiénes son los responsables y se ocupan de ello? ¿cuál es el tipo de abordaje sobre lo ambiental que subyace? ¿cuál es la gestión de lo ambiental en la escuela (prácticas institucionales sostenibles)? ¿es adecuada la formación docente? y, finalmente, ¿cómo se articula el problema de lo ambiental con otros temas escolares? Estos avances en materia educativa permitirán pensar cómo lograr aprendizajes significativos y las consecuentes acciones tendientes a revertir la tendencia negativa del impacto antropogénico en el ambiente.

\section{Referencias}

ANDERSON, L.; KRATHWOHL, D. A taxonomy for learning, teaching, and assessing: a revision of Bloom's taxonomy of educational objectives. New York: AddisonWesley, 2001.

ARGENTINA. Ley de educación nacional: ley no 26.206. Buenos Aires, 2006. Disponible en: <http://servicios.infoleg.gob.ar/infolegInternet/anexos/120000-124999/123542/ norma.htm>. Visitado en: 22 sep. 2016.

AUSUBEL, D. P. Adquisición y retención del conocimiento: una perspectiva cognitiva. Barcelona: Paidós Ibérica, 2002.

BLOOM, B. et al. Taxonomy of educational objectives: the classification of educational goals. New York: McKay, 1956. (Handbook I: cognitive domain).

BOIX MANSILLA, V.; GARDNER, H. Cuáles son las cualidades de la comprensión. In: WISKE, M. (Org.). La enseñanza para la comprensión: vinculación entre la investigación y la práctica. Buenos Aires: Paidós, 2005. p. 238-242. 
Percepciones de docentes y directores sobre los factores ...

BRAVO CHÁVEZ, W. Estudio de los conocimientos de los professores sobre educación ambiental. Revista de Didáctica Ambiental, v. 7, n. 9, p. 57-71, 2011. Disponible en: $<$ http://www.didacticaambiental.com/revista/numero9/conocimientos.pdf $>$. Visitado en: 22 sep. 2016.

BUENOS AIRES (Provincia). Subsecretaría de Educación. La educación ambiental en el marco de las transformaciones curriculares. Anales de la Educación Común, La Plata, v. 3, n. 8, p. 162-165, 2007. Disponible en: <http://servicios.abc.gov.ar/lainstitucion/ revistacomponents/revista/archivos/anales/numero08/archivosparadescargar/20_ed_ ambiental.pdf >. Visitado en: 15 nov. 2014.

CAMARENA GÓMEZ, B. O. La educación ambiental en el marco de los foros internacionales: una alternativa de desarrollo. Estudios Sociales, Hermosillo, v. 15, n. 28, p. 8-42, 2006. Disponible en: <http://www.redalyc.org/articulo.oa?id=41702801>. Visitado en: 21 sep. 2016.

CHROBAK, R. et al. Una aproximación a las motivaciones y actitudes del profesorado de enseñanza media de la provincia de Neuquén sobre temas de educación ambiental. Revista Electrónica de Enseñanza de las Ciencias, Vigo, v. 5, n. 1, p. 31-50, 2006. Disponible en: <http://reec.uvigo.es/volumenes/volumen5/ART3_Vol5_N1.pdf>. Visitado en: 21 sep. 2016.

CONDENANZA, L. M.; CORDERO, S. Educación ambiental y legislación educativa en Argentina: hacia un estado de la cuestión. Praxis Educativa, Santa Rosa, v. 17, n. 1, p. 47-55, 2013. Disponible en: <http://www.biblioteca.unlpam.edu.ar/pubpdf/praxis/ v17n1a05condenanza.pdf $>$. Visitado en: 21 sep. 2016.

CORBETTA, S.; SESSANO, P.; KRASMANSKI, M. Educación ambiental (EA), formación docente y TIC's, el desafío complejo de una triple articulación: hacia la definición de un espacio transversal. Revista de la Escuela de Ciencias de la Educación, Rosario, n. 7, 2012. Disponible en: <http://hdl.handle.net/2133/2944>. Visitado en: 21 sep. 2016.

DANIELSON, L. et al. Measuring the benefits of local public goods: environmental quality in Gaston County, North Carolina. Applied Economics, Abingdon, v. 27, n. 12, p. 12531260, 1995. Disponible en: <http://dx.doi.org/10.1080/00036849500000108>. Visitado en: 22 sep. 2016.

EDEL NAVARRO, R.; RAMÍREZ GARRIDO, M. S. Construyendo el significado del cuidado ambiental: un estudio de caso en educación secundaria. REICE: revista iberoamericana sobre calidad, eficacia y cambio en educación, Madrid, v. 4, n. 1, p. 52-70, 2006. Disponible en: < http://www.redalyc.org/pdf/551/55140106.pdf>. Visitado en: 15 nov. 2014.

ERICE, M. X. et al. Percepciones y valoraciones de actores sociales del sistema educativo sobre problemáticas ambientales en Mendoza, Argentina. Revista Electrónica de Investigación y Docencia, Jaén, n. 4, p. 55-78, 2010. Disponible en: <http://www.ujaen. es/revista/reid/revista/n4/REID4art3.pdf>. Visitado en: 21 sep. 2016. 
Saidón, M.; Claverie, J. A.

FARROKHIAN, F; HOSSEINPOUR, M.; SOLEIMANI, A. Assessment of elementary school teachers attitude towards major factors of environmental education in developing countries: case study in Iran. Journal of Applied Environmental and Biological Sciences, Cairo, v. 2, n. 11, p. 581-585, 2012.

FERNÁNDEZ CASO, M. V. et al. La imagen pública de la geografía: una indagación desde las visiones de profesores y padres de alumnos secundarios. Biblio 3w: revista bibliográfica de geografía y ciencias sociales, Barcelona, v. 15, n. 859, 2010. Disponible en: <www.ub.es/ geocrit/b3w-859.htm>. Visitado en: 21 sep. 2016.

FIEN, J. Teaching for a sustainable world: the environmental and development education project for teacher education. Environmental Education Research, Abingdon, v. 1, n. 1, p. 21-33, 1995. Disponible en: < http://dx.doi.org/10.1080/1350462950010102>. Visitado en: 21 sep. 2016.

FOGARTY, R.; STOEHR, J. Integrating curricula with multiple intelligences: teams, themes, and threads. Illinois: IRI/Skylight, 1995.

FRANZEN, A. Environmental attitudes in international comparison: an analysis of the ISSP surveys 1993 and 2000. Social Science Quarterly, Hoboken, v. 84, n. 2, p. 297-308, 2003.

FREIRE, P. Pedagogía de la autonomía: saberes necesarios para la práctica educativa. 2. ed. Buenos Aires: Siglo XXI, 1998.

GARCÍA COSTOYA, M.; ZENOBI, V. Educación ambiental: de la conservación a la formación para la ciudadanía. Buenos Aires: Dirección de Cultura y Enseñanza, Gobierno de la Ciudad de Buenos Aires, 2009.

GIL PÉREZ, D.; GAVIDIA CATALÁN, V.; FURIÓ MÁS, C. Problemáticas a las que la comunidad científica y la sociedad en general habrían de prestar una atención prioritaria. In: ROSÚA CAMPOS, J. L. et al. (Ed.). Universidad y sociedad para un futuro sostenible. Granada: Universidad de Granada, 1997. v. 1. p. 1-12.

GONZÁLEZ GAUDIANO, E.; ALBA CEBALLOS, A. Hacia unas bases teóricas de la educación ambiental. Enseñanza de las Ciencias, Barcelona, v.12, n. 1, p. 66-71, 1994. Disponible en: <http://www.raco.cat/index.php/Ensenanza/article/view/21335>. Visitado en: 21 sep. 2016.

GUREVICH, R. La cuestión ambiental y sus derivas educativas. In: Ambiente y educación: una apuesta al futuro. Buenos Aires: Paidós, 2011. p. 17-42.

GUTIÉRREZ PÉREZ, J.; POZO LLORENTE, T. Modelos teóricos contemporáneos y marcos de fundamentación de la educación ambiental para el desarrollo sostenible. Revista Iberoamericana de Educación, Madrid, v. 41, p. 21-68, 2006.

HEIMLICH, J. E. Environmental education evaluation: reinterpreting education as a strategy for meeting mission. Evaluation and Program Planning, Oxford, v. 33, n. 2, p. 180-185, 2010.

HICKS, D.; HOLDEN, C. Exploring the future: a missing dimension in environmental education. Environmental Education Research, Abingdon, v. 1, n. 2, 185-193, 1995. 
Percepciones de docentes y directores sobre los factores ...

LEFF, E. Sociología y ambiente: formación socioeconómica, racionalidad ambiental y transformaciones del conocimiento. In: (Coord.). Ciencias sociales y formación ambiental. Barcelona: Gedisa, 1994. p. 17-84.

MEIRA CARTEA, P. A.; CARIDE GÓMEZ, J. A. La geometría de la educación para el desarrollo sostenible, o la imposibilidad de una nueva cultura ambiental. Revista Iberoamericana de Educación, Madrid, n. 41, p. 103-116, 2006.

MORIN, E. Introducción al pensamiento complejo. 4. ed. Barcelona: Gedisa, 2001. NACIONES UNIDAS. Objetivos de desarrollo del milenio: informe de 2014. Nueva York, 2014. Disponible en: < http://www.un.org/es/millenniumgoals/pdf/mdg-report2014-spanish.pdf>. Visitado en: 15 nov. 2014.

Resolución aprobada por la asamblea general: 57/254. Decenio de las Naciones Unidas de la Educación para el desarrollo sostenible. [New York], 2002. (A/RES/57/254). Diponible en: <https://documents-dds-ny.un.org/doc/UNDOC/GEN/N02/556/15/ PDF/N0255615.pdf?OpenElement>. Visitado en: 19 sep. 2016.

NORTH, D. C. Instituciones, cambio institucional y desempeño económico. Mexico: Fondo de Cultura Económica, 1990.

PIAGET, J. La equilibración de las estructuras cognitivas: problema central del desarrollo. Madrid: Siglo XXI, 1978.

. Piaget's theory. In: MUSSEN, P. (Ed.). Carmichael's manual of child psychology. New York: John Wiley, 1983.

Recent studies in genetic epistemology. Cahiers de la Fondation Archives Jean Piaget, Genéve, n. 1, p. 3-7, 1980.

POTEL, D. Sondeo de percepción ambiental de los docentes de escuelas primarias de la Ciudad Autónoma de Buenos Aires. Buenos Aires: Defensoría del Pueblo, 2012.

POZO, J. I. Teorías cognitivas del aprendizaje. 5. ed. Madrid: Morata. 1997.

QUINTERO, S.; SAIDÓN, M.; BACHMAN, L. Qué saben y qué quieren saber los estudiantes secundarios sobre problemas ambientales. In: FERNÁNDEZ CASO, M. V.; GUREVICH, R. E. (Coord.). Didáctica de la geografía: prácticas escolares y formación de profesores. Buenos Aires: Biblos, 2014. p. 271-293.

RIVAROSA, A. et al. Pedagogía ambiental: territorio-identidad-emancipación. [S.l.: s.n.], [2009]. Disponible en: <http://www.deliberaweb.com/dades/documents/497/1272916925. pdf $>$. Visitado en: 22 sep. 2016.

SAIDÓN, M. Environmental returns to education: an application to waste management. E3 Journal of Environmental Research and Management, Milpitas, v. 4, n. 8, p. 302-309, 2012.

SAUVÉ, L. Uma cartografia das correntes em educação ambiental. In: SATO, M.; CARVALHO, I. C. M. (Org.). Educação ambiental: pesquisa e desafios. Porto Alegre: Artmed, 2005. p. 17-45. 
Saidón, M.; Claverie, J. A.

TILBURY, D. Environmental education for sustainability: defining the new focus of environmental education in the 1990s. Environmental Education Research, Abingdon, v. 1, n. 2, p. 195-212, 1995. Diponible en: <http://dx.doi.org/10.1080/1350462950010206>. Visitado en: 19 sep. 2016.

TRAVÉ GONZÁLEZ, G.; POZUELOS ESTRADA, F. J. Superar la disciplinariedad y la transversalidad simple: hacia un enfoque basado en la educación global. Investigación en la Escuela, Sevilla, n. 37, p. 5-11, 1999. Diponible en: <http://rabida.uhu.es/dspace/ handle/10272/10667>. Visitado en: 19 sep. 2016.

UNMÜßIG, B.; FATHEUER, T.; SACHS, W. Crítica a la economía verde: impulsos para un futuro social y ecológicamente justo. México: Fundación Heinrich Böll, [2012]. Diponible en: < https://mx.boell.org/sites/default/files/gruene_oekonomie_.pdf>. Visitado en: 19 sep. 2016.

Artigo recebido em 29/09/2015. Aceito em 12/04/2016.

Dirección para contato: Centro de Estudios de Estado y Sociedad Educación, Sánchez de Bustamante, 27, CABA 1173, Argentina. 